\title{
The Prognostic Impact of Left Ventricular Thrombus Resolution after Acute Coronary Syndrome and Risk Modulation via Anti-Thrombotic Treatment Strategies
}

\section{Felix Hofer}

Medical University of Vienna

Niema Kazem

Medical University of Vienna

Ronny Schweitzer

Medical University of Vienna

Patricia Horvat

Medical University of Vienna

Max-Paul Winter

Medical University of Vienna

Lorenz Koller

Medical University of Vienna

Christian Hengstenberg

Medical University of Vienna

Patrick Sulzgruber ( $\nabla$ patrick.sulzgruber@meduniwien.ac.at )

Medical University of Vienna

Alexander Niessner

Medical University of Vienna

\section{Research Article}

Keywords: Left ventricular thrombus, acute coronary syndrome, anti-thrombotic therapy

Posted Date: April 27th, 2021

DOI: https://doi.org/10.21203/rs.3.rs-439239/v1

License: (c) (1) This work is licensed under a Creative Commons Attribution 4.0 International License.

Read Full License 


\section{Abstract}

Background: Left ventricular thrombus (LVT) is a rare but dreaded complication during the acute phase of acute coronary syndrome (ACS). However, profound data on long-term outcome and associated antithrombotic treatment strategies of this highly vulnerable patient population are scarce in current literature.

Methods: Patients presenting with ACS were screened for presence of LVT and subsequently included within a prospective clinical registry. All-cause mortality and the composite of MACE and thromboembolic events were defined as primary and secondary endpoint.

Results: Within 43 patients presenting with LVT, thrombus resolution during patient follow-up was observed in 27 individuals (62.8\%). Patients that reached a resolution of LVT experienced lower incidence rates of death $(-23.9 \% ; p=0.022)$, MACE $(-37.8 \% ; p=0.005)$ and thrombo-embolic events $(-35.2 \% ; p=0.008)$. Even after adjustment for clinical variables, thrombus resolution showed an independent inverse association with all-cause death with an hazard ratio (HR) of $0.14(95 \% \mathrm{Cl}: 0.03-0.75 ; p=0.021)$ and as well as with MACE with a HR of $0.22(95 \% \mathrm{Cl}: 0.07-0.68 ; p=0.008)$ and thrombo-embolic events with a HR of 0.22 (95\% Cl: $0.06-0.75 ; p=0.015)$. Triple antithrombotic therapy (TAT) with ticagrelor/prasugrel showed a strong and independent association with thrombus resolution with an adjusted HR of $3.25(95 \% \mathrm{Cl}$ : $1.22-$ $8.68 ; p=0.019)$ compared to other strategies.

Conclusion: The presented data indicate a poor outcome of ACS patients experiencing LVT. In terms of a personalized risk stratification, thrombus resolution has a strong protective impact on both all-cause death and MACE with the potential to tailor treatment decisions - including an intensified anti-thrombotic treatment approach - in this patient population.

\section{Introduction}

Left ventricular thrombus (LVT) is a rare complication after acute coronary syndrome (ACS), especially occurring in patients presenting late with ST-elevation myocardial infarction (STEMI). ${ }^{1}$ Incidence rates differ among the observational studies from $1.6 \%$ up to $39 \%$ indicating that many LVT cases might remain undetected. ${ }^{2-7}$ This substantial variation in the incidence rate, is caused by varieties in the imaging modality used for diagnosis and the timing and frequency of screening. Additionally, the use of modern revascularization therapies has reduced the occurrence of LV-thrombus formation. ${ }^{2-7}$ While the prognosis of patients presenting with LVT after ACS has been controversially discussed, it seems intuitive that individuals without thrombus resolution have an increased risk for cardiovascular events and mortality. Thrombus formation is significantly associated with anterior myocardial infarction and confers an increased risk for thromboembolic events (mostly cerebrovascular). ${ }^{8-10}$ In the pre-thrombolytic era, these complications were described in approximately $10 \%$ of cases, whereas in the era of thrombolytic therapy embolic events occurred in 2-3\% of cases. ${ }^{8-10}$ Until now there is scarce evidence regarding the incidence of embolic events in patients treated by primary percutaneous coronary intervention (PCl) that 
receive dual anti-platelet therapy (DAPT) or even dual or triple anti-thrombotic therapy (DAT,TAT). However, optimal pharmacological therapy - used to reduce complications of LVT - remains challenging. While patients post myocardial infarction (MI) require DAPT for reduction of atherothrombotic risk, they also need oral anticoagulation (OAC) in case of LVT formation for reduction of related complications, with subsequent high risk of bleeding. ${ }^{11}$ Considering a strong impact of LVT on patient outcome and the notion that many LVTs remain undetected in clinical practice, patient characteristics that help to identify ACS individuals at risk for the development of LVT with an adverse outcome should be considered in terms of a personalized secondary prevention. However, profound data on long-term outcome of this highly vulnerable patient population are scarce in current literature. Therefore, we aimed to investigate the impact of LVT resolution and associated anti-thrombotic treatment strategies on patient's outcome from a long-term perspective.

\section{Methods}

\section{Study population and patient selection}

Patients presenting with ACS $(n=2011)$ who underwent treatment at the Vienna General Hospital, a university affiliated tertiary care center with a high-volume cardiac catheterization unit in the time period between 01/2015-09/2019 were screened for presence of LVT. Out of the source population a total of 52 patients (2.6\%) presented with LVT after MI. Six individuals died before hospital discharge and 3 did not receive follow-up imaging and were subsequently excluded for the final analysis - resulting in a total study population of 43 patients for the present long-term analysis. All patients were older than 18 years. The study was conducted in accordance to the current criteria of the Declaration of Helsinki and was approved by the ethics committee of the Medical University of Vienna (1702/2019). After completion of follow-up, the study population was stratified in patients with thrombus resolution and individuals without thrombus resolution.

\section{Data acquisition and patient follow-up}

Patient-relevant characteristics were assessed via the patients' electronic medical records of the Vienna General Hospital, as well during a standardized follow-up procedure. Data assessment was performed by specially trained chart reviewers that inserted predefined patient characteristics into a record abstraction form for further analysis of the registry at the time of hospitalization and re-evaluation during the entire hospitalization. Discharge letters of all participants were screened for the antithrombotic treatment approach at the time of discharge.

Patients were invited to the local department for screening of thrombus resolution. The presence of LVT was validated by transthoracic echocardiography (TTE) and/or cardiac magnetic resonance tomography (CMR) of all enrolled individuals. All TTE or CMR were reviewed by at least two cardiologists.

Clinically relevant data, including the anti-thrombotic treatment and adherence to medication was assessed during the follow-up visit. Thrombus resolution was defined as lack of evidence of thrombus 
mass in follow-up contrast TTE or CMR.

\section{Endpoint Definition}

All-cause death was chosen as primary study endpoint. The composite of major adverse cardiac events (MACE), defined as a non-fatal myocardial infarction, non-fatal stroke, and cardiovascular death as well as thrombo-embolic events (systemic embolism, pulmonary embolism) were chosen as secondary endpoints and assessed during follow-up. Bleeding events were defined referring to the definition of the Bleeding Academic Research Consortium (BARC). The patients' cause and date of death was assessed by screening the national registry of death until December 2019 via the Austrian Registry of Death (Statistics Austria, Vienna, Austria). Causes of death were defined according to the International Statistical Classification of Disease and Related Health Problems 10th Revision.

\section{Statistical Analysis}

Continuous data are presented as median and the respective interquartile range and analyzed using Mann Whitney $U$ test. Categorical parameters are presented as counts and percentages and analyzed using Chi-square test. Univariate and multivariate Cox proportional hazard models were applied to assess the influence of thrombus resolution on primary and secondary endpoints and to assess the impact of a TAT with newer $\mathrm{P}_{2} \mathrm{Y}_{12}$ antagonists on LVT resolution. Results were presented as hazard ratio (HR) and the respective $95 \%$ confidence interval $(\mathrm{Cl})$. A three-step adjustment approach was followed within the multivariate regression model including comprehensive adjustment for patient characteristics (= Model 1: age and sex), clinical presentation (= Model 2: STEMI and heart failure) and laboratory values (= Model 3 : Nt-proBNP and creatinine kinase [CK]).

Continuous variables were log-transformed prior to inclusion in the regression analysis. Kaplan Maier charts were plotted to graphically illustrate the impact of LVT resolution on all-cause death, MACE and thrombo-embolic events and compared using log-rank test. Statistical significance was defined by twosided p-values $<0.05$. Statistical analyses were performed using SPSS 26.0 (IBM SPSS, NY, USA).

\section{Results}

Detailed baseline characteristics for the study population presenting with LVT $(n=43)$, stratified in individuals with thrombus resolution and without thrombus resolution are summarized in Table 1.

In short, the present study population (median age: 63 years [IQR 58-69]; 88.4\% male gender) covered a representative number of participants presenting with STEMI $(n=26 ; 60.5 \%)$ and all patients developed LVT after anterior wall infarction. The remaining $39.5 \%$ of patients presented with non-ST-elevation myocardial infarction (NSTEMI). Patients in the thrombus resolution group trended to be younger $(\mathrm{p}=$ 0.074). In $97.7 \%$ of cases LVT was diagnosed via TTE and in $2.3 \%$ via CMR. The median time of thrombus detection after the acute event was 5 days [IQR 3-15]. The median time between thrombus detection and the first follow up imaging was 14 weeks [IQR 6-22]. 
Comparing characteristics of patients with thrombus resolution and without thrombus resolution we observed balanced frequencies of cardiovascular risk factors, such as diabetes mellitus $(p=0.610)$, hyperlipidemia $(p=0.258)$ and hypertension $(p=0.746)$. Established risk factors for the development of LVT - such as reduced left ventricular ejection fraction (48.8\%), anterior wall infarction (100\%) and elevated Nt-proBNP values (median: 1526.0 [IQR 609.3-7012.3]) reflecting cardiac strain were observed with high frequencies within the study population. However, we did not observe any significant association with thrombus resolution except for a trend of higher Nt-proBNP levels $(p=0.089)$. Detailed baseline echocardiographic parameters are shown in table 2 . In short, severe left ventricular dysfunction was common (median left ventricular ejection fraction (LVEF) 36.0\% [IQR 33.0-45.0]) with no differences between both groups $(p=0.908)$. Left ventricular aneurysms were found in $27.9 \%$ of patients $(n=12)$ without any group differences $(p=0.286)$. Median time of thrombus resolution was 14 weeks [IQR 6-23].

\section{Follow-up and outcome analysis}

After a median follow-up time of 108 weeks [IQR 68-173], $16.3 \%$ of patients died ( $n=7)$, with $31.3 \%$ of individuals $(n=5)$ in the no LVT resolution subgroup and $7.4 \%(n=2)$ in the LVT resolution subgroup, respectively $(p=0.022)$ (table 3$)$. Cardiovascular death occurred in $9.3 \%$ of patients with LVT $(n=4)$ with a trend for lower event rate in the LVT resolution group (3.7\% vs $18.8 \% ; p=0.062)$. In total, MACE occurred in $32.6 \%(n=14)$ of cases with a LVT, resulting in a significantly lower rate of MACE in the resolution group compared to the no resolution group $(18.5 \%$ vs $56.3 \% ; p=0.005)$. Thrombo-embolic events occurred in $27.9 \%$ of cases $(n=12)$, including $14.8 \%(n=4)$ in the LVT resolution subgroup and $50.0 \%(n=8)$ in the no LVT resolution subgroup $(p=0.008)$. Major bleeding events occurred in $9.3 \%$ of individuals $(n=4)$ presenting with LVT, without significant subgroup differences $(p=0.296)$. (table 3 ) LVT resolution proved to be inversely associated with long-term mortality, presenting with a crude HR of 0.18 (95\% Cl: $0.03-0.93$; $p=0.041$ ). Three different multivariate models (1. patient characteristics, 2 . clinical presentation and 3. laboratory values) were used to analyze whether the prognostic value of LVT was independently associated with mortality, MACE and thrombo-embolic events (Table 4). Within the multivariate model 2, LVT resolution remained inversely associated with long-term mortality with an adjusted HR of 0.14 ( $95 \%$ Cl: $0.03-0.75 ; p=0.021$ ) (table 4 ).

In addition, LVT resolution was also associated with a significant lower risk of MACE with a crude HR of 0.26 (95\% Cl: $0.09-0.77 ; p=0.015)$ and thrombo-embolic events with a crude HR of 0.23 (95\% Cl: $0.07-$ $0.78 ; p=0.018)$. LVT resolution remained inversely associated with MACE, after adjustment for model 1 (adj. HR of 0.24 (95\% Cl: 0.08-0.71); $p=0.010$ ) and model 2 (adj. HR of 0.22 (95\% Cl: 0.07-0.68); $p=$ 0.008) and thrombo-embolic events after adjustment for model 1 (adj. HR of 0.21 (95\% Cl: 0.06-0.72); $p=$ 0.013 ), model 2 (adj. HR of 0.22 (95\% Cl: 0.06-0.75); $p=0.015)$ and model 3 (adj. HR of 0.24 (95\% Cl: $0.07-0.86) ; p=0.029$ ). (table 4)

Event rates for all-cause death, MACE and thrombo-embolic events at 1 year were $3.7 \%, 14.8 \%$, and $11.1 \%$ in the thrombus resolution group, compared to $25.0 \%, 43.8 \%$ and $43.8 \%$ in the no thrombus resolution 
group respectively.

The Kaplan Meier survival plot and log-rank test indicated a higher risk of long-term death $(p=0.022)$, MACE $(p=0.009)$ and thrombo-embolic events $(p=0.010)$ for individuals without LVT resolution as compared to patients with LVT resolution. (see figure 1 ).

\section{Anti-thrombotic treatment strategies}

Considering anti-thrombotic treatment strategies, we observed that all patients received OAC including 10 patients (23.3\%) receiving non-vitamin-K oral anticoagulants (NOACs) and 33 patients $(76.7 \%)$ Vitamin $\mathrm{K}$ antagonists (VKA) respectively immediately after diagnosis. Median duration of anticoagulation therapy was 24 weeks [12-72] without significant difference between both groups. The fraction of individuals receiving DAT or TAT did also not differ significantly between both groups $(p=0.137)$. However, the use of TAT with a more potent $\mathrm{P}_{2} \mathrm{Y}_{12}$ inhibitor such as ticagrelor or prasugrel was observed only in the thrombus resolution group ( $38.1 \%$ vs. $0 \% ; p=0.031)$. Median duration of antiplatelet therapy with ticagrelor/prasugrel was 12 months, with a similar duration of 12 months for antiplatelet therapy with clopidogrel - there was no significant differences between both groups. Most importantly TAT with either ticagrelor or prasugrel showed a strong and independent association with thrombus resolution with a crude HR of 3.67 (95\% Cl: 1.53-8.81; $p=0.004)$. Notably, the prognostic impact remained stable after adjustment for model 1 (adj. HR of 3.69 (95\% Cl: 1.53-8.91; $p=0.004$ ), model 2 (adj. HR of 3.25 (95\% Cl: $1.22-8.68 ; p=0.019)$ and model 3 (adj. HR of 2.69 (95\% Cl: 1.10-6.58; $p=0.030)$. (table 5)

\section{Discussion}

The current analysis is - to the best of our knowledge - the first in literature that investigated the impact of LVT resolution after ACS on cardiovascular events and mortality. The present data illustrates that LVT resolution was independently associated with a favorable long-term outcome and survival free of MACE and thrombo-embolic events. In addition, our data indicates that TAT with potent P2Y 12 antagonists might be considered in patients with LVT.

Within the present investigation we observed an incidence rate of LVT after ACS of $2.5 \%$. Reported incidence rates vary from $1.6 \%$ up to $39 \% .{ }^{2-7}$ The principal cause of these variations is rooted in the use of modern revascularization therapies ${ }^{2-7}$ Recent studies reported incidence rates below $10 \%$, whereas in the pre-thrombolytic era LVT formation was observed in up to $39 \%$ of patients after MI. 7,12,13 Furthermore, different imaging modalities and the timing and frequency of screening may affect the incidence rate. ${ }^{2-7}$

Within our study we observed a high prevalence of established risk factors for LVT as well as high values of Nt-proBNP, Troponin T and CK indicating extensive tissue damage and scar formation. Functional myocardial tissue loss leading to left ventricular wall motion abnormalities and reduced cardiac output represents a key factor in the development of LVT. ${ }^{14-16}$ All patients developed LVT after anterior wall 
infarction which is in line with previous studies showing a significant association between LVT formation and left anterior descending artery as the culprit lesion. ${ }^{7}$ Our data also suggest decreased thrombus resolution rates in patients with increased Nt-proBNP levels indicating decreased myocardial function.

Optimal pharmacological therapy in this highly vulnerable patient population - used to reduce complications of LVT - remains challenging. Within the present investigation VKA is the primary anticoagulant used with low prescription rates of NOACs, which is probably caused by the lack of evidence in the treatment of LVT. ${ }^{11}$ Furthermore, we observed that one third of patients did not achieve LVT resolution, despite additional anticoagulation. Our data indicates that the current antithrombotic strategy needs to be improved to reduce associated clinical complications. In line with our findings, a recently published study by Lattuca and colleagues observed thrombus regression in $62 \%$ of patients with LVT treated with OAC. ${ }^{17}$ A further study of 92 LVT patients treated with VKA demonstrated that thrombus resolution was dependent on time spent within the therapeutic range. ${ }^{18}$ However, the narrow therapeutic window of VKAs poses a major problem in therapy necessitating frequent monitoring and dosage adjustments. Treatment with NOACs cannot be recommended at this time due to a lack of robust evidence, despite similar thrombus resolution rates compared to VKAs within our study and in nonrandomized trials. ${ }^{11,17,18}$ of note, a recently published study by Robinson et al. indicates that anticoagulation with NOACs was associated with a higher risk of ischemic stroke and systemic emboli compared with warfarin treatment in patients with LVT. ${ }^{19}$ However, these results are limited by a lack of randomization and by the retrospective nature of this analysis. ${ }^{19}$

Considering a similar but not identical effect of NOACs and in particular Xa-inhibitors in resolution of left atrial thrombus (LAT) and LVT resolution, randomized studies assessing LAT resolution provide us some evidence on the potential effect of LVT resolution. In the recently published EMANATE trial, similar LAT resolution rates were reported in patients receiving apixaban (52\%) vs. patients receiving heparin/VKA (58\%). ${ }^{20}$ The mean follow-up imaging period was 5 weeks after diagnosis compared to a median time of 14 weeks in our trial. ${ }^{20}$ Another study by Lip et al. showed a $60.4 \%$ LAT resolution/reduction rate in patients with atrial fibrillation or atrial flutter treated with rivaroxaban. ${ }^{21}$ Nevertheless, a specific effect of NOACs on LVT resolution needs to be proven in future randomized controlled trials (RCT) with a particular focus on the required treatment period. Currently, ESC guidelines recommend anticoagulation with a VKA for up to 6 months in the presence of a LVT after ACS. ${ }^{11}$

Surprisingly, $26.7 \%$ of patients receiving TAT received ticagrelor/prasugrel as a part of the regimen. Further analysis suggests that TAT with a more potent $\mathrm{P} 2 \mathrm{Y}_{12}$ antagonist is associated with a markedly increased likelihood of thrombus resolution. However, a meta-analysis of several randomized controlled clinical trials (WOEST, PIONEER, RE DUAL, ENTRUST-AF PCI and AUGUSTUS) investigating the efficacy and safety of DAT versus TAT in patients with atrial fibrillation undergoing coronary intervention has shown a significantly reduced bleeding risk in patients with DAT compared to TAT and similar efficacy in preventing ischemic events. ${ }^{22}$ In conclusion, TAT with prasugrel/ticagrelor might be an attractive option 
in patients with persistent LVT after conventional therapy and low bleeding risk but future studies are needed to evaluate the bleeding risk in this population.

With respect to clinical endpoints, our data clearly demonstrated a lower risk for MACE, thrombo-embolic events, and all-cause death after LVT resolution. Consistent with our findings, Lattuca and colleagues showed that the clinical prognosis of patients with LVT is poor with a very high risk of major cardiovascular events and mortality. Furthermore, they could assess that LVT resolution, obtained with different anticoagulant strategies, was associated with reduced mortality, which may serve as a basis for using LVT resolution as a surrogate endpoint in future randomized controlled trials. ${ }^{19}$

\section{Limitations}

Despite the extended follow-up, several limitations should be addressed. Firstly, patients were not followed using a standardized follow-up and follow-up imaging was part of usual care.

A further limitations of the present analysis represent its single center setting and the low sample size. However, considering the rare occurrence of LVT after MI and long screening period, we obtained a clinically relevant sample size for the present investigation.

\section{Conclusion}

The presented data clearly highlighted the poor outcome of ACS patients experiencing LVT. In terms of a personalized risk stratification, the prognostic value of thrombus resolution on MACE, all-cause death and thrombo-embolic events can reasonably be considered for risk assessment and treatment decisions in this highly vulnerable patient population. Considering the observation that TAT with more potent P2Y 12 antagonists was associated with a treatment benefit, an intensified anti-thrombotic treatment approach might be taken into account in patients with persistent LVT and low bleeding risk.

\section{Declarations}

\section{Compliance with Ethical Standards}

Due to the observational character of the study, patient informed consent was not required and it was therefore waved by the ethics committee of the Medical University of Vienna.

The study protocol complies with the declaration of Helsinki and was approved by the local ethics committee of the Medical University of Vienna (1702/2019).

\section{Funding Information}

This research did not receive any specific grant from funding agencies in the public, commercial, or notfor-profit sectors. 


\section{Conflict of interest}

Felix Hofer: none

Niema Kazem: none

Ronny Schweitzer: none

Patricia Horvat: none

Max-Paul Winter: none

Lorenz Koller: none

Christian Hengstenberg: none

Patrick Sulzgruber: grants from Daiichi Sankyo and grants from Boehringer-Ingelheim outside the submitted work

Alexander Niessner: personal fees from Bayer, personal fees from BMS, grants and personal fees from Boehringer Ingelheim, grants and personal fees from Daiichi Sankyo and personal fees from Pfizer outside the submitted work

\section{Authors contribution}

AN, PS and FH contributed to the conception or design of the work. FH, PH, RS and MPW contributed to the acquisition, analysis, or interpretation of data for the work. FH drafted the manuscript. AN, NK, LK and $\mathrm{CH}$ critically revised the manuscript. All gave final approval and agree to be accountable for all aspects of work ensuring integrity and accuracy.

Acknowledgements

None

Availability of data and material

Data will be provided upon request

\section{References}

1. Sanchis-Gomar, F., Perez-Quilis, C., Leischik, R. \& Lucia, A. Epidemiology of coronary heart disease and acute coronary syndrome. Annals of translational medicine. 4, 256 https://doi.org/10.21037/atm.2016.06.33 (2016). 
2. McCarthy, C. P. et al. Left Ventricular Thrombus After Acute Myocardial Infarction: Screening, Prevention, and Treatment. JAMA cardiology. https://doi.org/10.1001/jamacardio.2018.1086 (2018).

3. Asinger, R. W., Mikell, F. L., Elsperger, J. \& Hodges, M. Incidence of left-ventricular thrombosis after acute transmural myocardial infarction. Serial evaluation by two-dimensional echocardiography. The New England journal of medicine. 305, 297-302 https://doi.org/10.1056/nejm198108063050601 (1981).

4. Domenicucci, S. et al. Early appearance of left ventricular thrombi after anterior myocardial infarction: a marker of higher in-hospital mortality in patients not treated with antithrombotic drugs. European heart journal. 11, 51-58 (1990).

5. Keren, A. et al. Natural history of left ventricular thrombi: their appearance and resolution in the posthospitalization period of acute myocardial infarction. Journal of the American College of Cardiology. 15, 790-800 (1990).

6. Kontny, F. et al. Left ventricular thrombosis and arterial embolism after thrombolysis in acute anterior myocardial infarction: predictors and effects of adjunctive antithrombotic therapy. European heart journal. 14, 1489-1492 (1993).

7. Robinson, A. A., Jain, A., Gentry, M. \& McNamara, R. L. Left ventricular thrombi after STEMI in the primary PCl era: A systematic review and meta-analysis. International journal of cardiology. 221, 554-559 https://doi.org/10.1016/j.ijcard.2016.07.069 (2016).

8. Johannessen, K. A., Nordrehaug, J. E., von der Lippe, G. \& Vollset, S. E. Risk factors for embolisation in patients with left ventricular thrombi and acute myocardial infarction. British heart journal. 60 , 104-110 (1988).

9. Meltzer, R. S., Visser, C. A. \& Fuster, V. Intracardiac thrombi and systemic embolization. Annals of internal medicine. 104, 689-698 (1986).

10. Haugland, J. M., Asinger, R. W., Mikell, F. L., Elsperger, J. \& Hodges, M. Embolic potential of left ventricular thrombi detected by two-dimensional echocardiography. Circulation. 70, 588-598 (1984).

11. Ibanez, B. et al. 2017 ESC Guidelines for the management of acute myocardial infarction in patients presenting with ST-segment elevation: The Task Force for the management of acute myocardial infarction in patients presenting with ST-segment elevation of the European Society of Cardiology (ESC). European heart journal. 39, 119-177 https://doi.org/10.1093/eurheartj/ehx393 (2018).

12. Kalra, A. \& Jang, I. K. Prevalence of early left ventricular thrombus after primary coronary intervention for acute myocardial infarction. Journal of thrombosis and thrombolysis. 10, 133-136 https://doi.org/10.1023/a:1018710425817 (2000).

13. Zielinska, M., Kaczmarek, K. \& Tylkowski, M. Predictors of left ventricular thrombus formation in acute myocardial infarction treated with successful primary angioplasty with stenting. The American journal of the medical sciences. 335, 171-176 https://doi.org/10.1097/MAJ.0b013e318142be20 (2008).

14. Merlini, P. A. et al. Persistent activation of coagulation mechanism in unstable angina and myocardial infarction. Circulation. 90, 61-68 https://doi.org/10.1161/01.cir.90.1.61 (1994). 
15. Stein, B. \& Fuster, V. Antithrombotic therapy in acute myocardial infarction: prevention of venous, left ventricular and coronary artery thromboembolism. The American journal of cardiology $64,33 \mathrm{~b}-40 \mathrm{~b}$, doi:10.1016/s0002-9149(89)80008-6 (1989).

16. Taruya, A. et al. Left ventricular ball-like thrombus after acute myocardial infarction with essential thrombocythemia. Journal of Cardiology Cases. 10, 1-3

https://doi.org/10.1016/j.jccase.2014.01.005 (2014).

17. Lattuca, B. et al. Antithrombotic Therapy for Patients With Left Ventricular Mural Thrombus. Journal of the American College of Cardiology. 75, 1676-1685 https://doi.org/10.1016/j.jacc.2020.01.057 (2020).

18. Maniwa, N. et al. Anticoagulation combined with antiplatelet therapy in patients with left ventricular thrombus after first acute myocardial infarction. European heart journal. 39, 201-208 https://doi.org/10.1093/eurheartj/ehx551 (2018).

19. Robinson, A. A. et al. Off-label Use of Direct Oral Anticoagulants Compared With Warfarin for Left Ventricular Thrombi. JAMA cardiology. https://doi.org/10.1001/jamacardio.2020.0652 (2020).

20. Ezekowitz, M. D. et al. Apixaban compared to heparin/vitamin $\mathrm{K}$ antagonist in patients with atrial fibrillation scheduled for cardioversion: the EMANATE trial. European heart journal. 39, 2959-2971 https://doi.org/10.1093/eurheartj/ehy148 (2018).

21. Lip, G. Y. et al. Left atrial thrombus resolution in atrial fibrillation or flutter: Results of a prospective study with rivaroxaban (X-TRA) and a retrospective observational registry providing baseline data (CLOT-AF). American heart journal. 178, 126-134 https://doi.org/10.1016/j.ahj.2016.05.007 (2016).

22. Haller, P. M. et al. Bleeding and ischaemic outcomes in patients treated with dual or triple antithrombotic therapy: systematic review and meta-analysis. European heart journal. Cardiovascular pharmacotherapy. 5, 226-236 https://doi.org/10.1093/ehjcvp/pvz021 (2019).

\section{Tables}

Tables 1: Baseline characteristics 


\begin{tabular}{|c|c|c|c|c|}
\hline & $\begin{array}{l}\text { Overall } \\
(n=43)\end{array}$ & $\begin{array}{l}\text { No } \\
\text { Resolution } \\
\qquad(n=16)\end{array}$ & $\begin{array}{l}\text { Resolution } \\
(\mathrm{n}=27)\end{array}$ & $\begin{array}{l}\mathrm{P} \text { - } \\
\text { value }\end{array}$ \\
\hline \multicolumn{5}{|l|}{ Clinical Presentation } \\
\hline Age, years (IQR) & $63(58-69)$ & $68(61-72)$ & $62(56-67)$ & 0.074 \\
\hline Gender (male), n (\%) & $38(88.4)$ & $14(87.5)$ & $24(88.9)$ & 0.892 \\
\hline $\mathrm{BMI}, \mathrm{kg} / \mathrm{m}^{2}$ (IQR) & $\begin{array}{l}26.2(24.2- \\
29.5)\end{array}$ & $26.1(24.1-29.2)$ & $\begin{array}{l}26.3(24.2- \\
30.2)\end{array}$ & 0.756 \\
\hline $\mathrm{PCl}, \mathrm{n}(\%)$ & $33(76.7)$ & $10(62.5)$ & $23(85.2)$ & 0.093 \\
\hline STEMI, n (\%) & $26(60.5)$ & $8(50.0)$ & $18(66.7)$ & 0.289 \\
\hline Anterior wall infarction, $\mathrm{n}(\%)$ & $43(100)$ & $16(100)$ & $27(100)$ & 1.000 \\
\hline Systolic BP, mmHg (IQR) & $\begin{array}{l}135.0 \\
(115.8 .-149.3)\end{array}$ & $\begin{array}{l}135.5(109.8- \\
148.3)\end{array}$ & $\begin{array}{l}135.0(116.0- \\
150.0)\end{array}$ & 0.836 \\
\hline Diastolic BP, mmHg (IQR) & $\begin{array}{l}74.0(68.0- \\
90.0)\end{array}$ & $74.0(66.5-89.0)$ & $\begin{array}{l}74.0(69.5- \\
90.0)\end{array}$ & 0.785 \\
\hline LVEF <50\% (\%) & $21(48.8)$ & $7(43.8)$ & $14(51.9)$ & 0.612 \\
\hline \multicolumn{5}{|l|}{ Comorbidities } \\
\hline Diabetes, n (\%) & $7(16.3)$ & $2(12.5)$ & $5(18.5)$ & 0.610 \\
\hline Hyperlipidemia, n (\%) & $21(48.8)$ & $6(37.5)$ & $15(55.6)$ & 0.258 \\
\hline Hypertension, n (\%) & $31(72.1)$ & $12(75.0)$ & $19(70.4)$ & 0.746 \\
\hline Nicotine, n (\%) & $22(51.2)$ & $6(37.5)$ & $16(59.3)$ & 0.173 \\
\hline Prior MI. n (\%) & $15(34.9)$ & $5(31.3)$ & $10(37.0)$ & 0.704 \\
\hline \multicolumn{5}{|l|}{ Medication } \\
\hline Beta Blockers, n (\%) & $41(95.3)$ & $16(100)$ & $25(92.6)$ & 0.271 \\
\hline ACEI, n (\%) & $31(72.1)$ & $12(75.0)$ & $19(70.4)$ & 0.746 \\
\hline ATI, n (\%) & $8(18.6)$ & $1(6.3)$ & $7(25.9)$ & 0.113 \\
\hline \multicolumn{5}{|c|}{ Antithrombotic treatment approach } \\
\hline Acetylsalicylic acid, n (\%) & $43(100)$ & $16(100)$ & $27(100)$ & 1.000 \\
\hline Clopidogrel, n (\%) & $22(51.2)$ & $9(56.3)$ & $13(48.1)$ & 0.612 \\
\hline Ticagrelor/Prasugrel, n (\%) & $8(7.0)$ & $0(-)$ & $8(29.6)$ & 0.016 \\
\hline
\end{tabular}




\begin{tabular}{|c|c|c|c|c|}
\hline DAT, n (\%) & $13(30.2)$ & $7(43.8)$ & $6(22.2)$ & 0.137 \\
\hline TAT, n (\%) & $30(69.8)$ & $9(56.3)$ & 21 (77.8) & 0.137 \\
\hline TAT with clopidogrel, n(\%) & $22(73.3)$ & $9(100)$ & $13(61.9)$ & 0.031 \\
\hline $\begin{array}{l}\text { Duration of clopidogrel (months), } \\
\text { median (IQR) }\end{array}$ & $12(12-12)$ & $12(12-12)$ & $12(12-12)$ & 1.000 \\
\hline TAT with ticagrelor/prasugrel, $n$ (\%) & $8(26.7)$ & $0(-)$ & $8(38.1)$ & 0.031 \\
\hline $\begin{array}{l}\text { Duration of ticagrelor/prasugrel } \\
\text { (months) median (IQR) }\end{array}$ & $12(12-12)$ & - & $12(12-12)$ & NA \\
\hline VKA, n (\%) & $33(76.7)$ & $13(81.3)$ & $20(74.1)$ & 0.595 \\
\hline NOAC n (\%) & $10(23.3)$ & $3(18.8)$ & $7(25.9)$ & 0.595 \\
\hline $\begin{array}{l}\text { Duration of OAK (months) median } \\
\text { (IQR) }\end{array}$ & $24(12-72)$ & $24(18-64)$ & $12(12-84)$ & 0.780 \\
\hline \multicolumn{5}{|l|}{ Laboratory variables } \\
\hline NTproBNP, pg/ml (IQR) & $\begin{array}{l}1526.0 \\
(609.3- \\
7012.3)\end{array}$ & $\begin{array}{l}2945.0(1040.3- \\
20355.0)\end{array}$ & $\begin{array}{l}1308.0 \\
(401.8- \\
4284.8)\end{array}$ & 0.089 \\
\hline Troponin T max., $\mu \mathrm{g} / \mathrm{ml}$ median (IQR) & $\begin{array}{l}2339.5 \\
(224.5- \\
5828.0)\end{array}$ & $\begin{array}{l}1324.5(44.5- \\
3583.0)\end{array}$ & $\begin{array}{l}3502.0 \\
(548.8- \\
7455.5)\end{array}$ & 0.431 \\
\hline CK, U/I median (IQR) & $\begin{array}{l}986.0(119.5- \\
2906.5)\end{array}$ & $\begin{array}{l}222.0(68.0- \\
1545.0)\end{array}$ & $\begin{array}{l}1355.0 \\
(281.5- \\
3431.3)\end{array}$ & 0.066 \\
\hline CK-MB, U/I median (IQR) & $\begin{array}{l}191.5(49.0- \\
283.0)\end{array}$ & $\begin{array}{l}77.0(26.0- \\
465.0)\end{array}$ & $\begin{array}{l}200(61.0- \\
296.0)\end{array}$ & 0.455 \\
\hline
\end{tabular}

Categorical data are presented as counts and percentages and analyzed using Chi-square-test. Continuous data are presented as median and the respective interquartile range and analyzed using Mann Whitney $\mathrm{U}$ test. $\mathrm{ACEI}=$ Angiotensin converting enzyme inhibitor, $\mathrm{AF}=$ Atrial fibrillation, $\mathrm{ATI}=$ Angiotensin II receptor inhibitor, $\mathrm{BP}=$ Blood pressure, $\mathrm{BMI}=$ Body-mass index, $\mathrm{CK}=$ Creatinine kinase DAPT = Dual antiplatelet therapy, DAT $=$ Dual anti-thrombotic therapy, INR $=$ International normalized ratio, IQR = Interquartile range, $\mathrm{LVEF}=$ Left ventricular ejection fraction, $\mathrm{MI}=$ Myocardial infarction, NOAC $=$ Non-vitamin-K anticoagulant, NT-proBNP $=\mathrm{N}$-terminal pro b-type natriuretic peptide, $\mathrm{PCl}=$ Percutaneous coronary intervention, STEMI = ST elevation myocardial infarction, TAT = Triple anti-thrombotic therapy, VKA = Vitamin-K antagonist.

Table 2: Baseline Echocardiographic Parameters 


\begin{tabular}{|c|c|c|c|c|}
\hline & $\begin{array}{l}\text { Overall } \\
(n=43)\end{array}$ & $\begin{array}{l}\text { No } \\
\text { Resolution } \\
(n=16)\end{array}$ & $\begin{array}{l}\text { Resolution } \\
(n=27)\end{array}$ & $\begin{array}{l}\mathrm{P}- \\
\text { value }\end{array}$ \\
\hline Left ventricular ejection fraction, \% & $\begin{array}{l}36.0(33.0- \\
45.0)\end{array}$ & $\begin{array}{l}39.0(22.0- \\
50.0)\end{array}$ & $\begin{array}{l}36.0(35.0- \\
40.0)\end{array}$ & 0.908 \\
\hline $\begin{array}{l}\text { End-diastolic left ventricular diameter, } \\
\mathrm{mm}\end{array}$ & $\begin{array}{l}49.0(43.0- \\
55.0)\end{array}$ & $\begin{array}{l}49.0(43.0- \\
57.0)\end{array}$ & $\begin{array}{l}48.0\left(42.8^{-}\right. \\
53.3)\end{array}$ & 0.586 \\
\hline $\begin{array}{l}\text { End-diastolic right ventricular } \\
\text { diameter, } \mathrm{mm}\end{array}$ & $\begin{array}{l}33.0(29.0- \\
36.0)\end{array}$ & $\begin{array}{l}35.0(29.5- \\
40.0)\end{array}$ & $\begin{array}{l}31.0(28.0- \\
34.0)\end{array}$ & 0.617 \\
\hline $\begin{array}{l}\text { Interventricular septum thickness, cm } \\
\text { Left ventricular thrombus }\end{array}$ & $1.3(1.2-1.4)$ & $\begin{array}{l}13.0(11.3- \\
14.0)\end{array}$ & $\begin{array}{l}13.0(11.5- \\
14.5)\end{array}$ & \\
\hline Area, $\mathrm{cm}^{2}$ & $2.1(0.9-4.2)$ & $3.1(0.8-5.0)$ & $2.1(1.6-4.1)$ & 0.956 \\
\hline Volume, $\mathrm{cm}^{3}$ & $1.7(1.1-4.2)$ & $2.1(0.4-4.8)$ & $1.7(1.1-4.2)$ & 0.977 \\
\hline Apical thrombus, $n$ (\%) & $43(100)$ & $16(100)$ & $27(100)$ & 1.000 \\
\hline Left ventricular aneurysm, $n(\%)$ & $12(27.9)$ & $6(37.5)$ & $6(22.2)$ & 0.286 \\
\hline
\end{tabular}

Categorical data are presented as counts and percentages and analyzed using Chi-square-test.

Continuous data are presented as median and the respective interquartile range and analyzed using Mann Whitney U test.

Table 3: Cardiovascular Events

\begin{tabular}{|c|c|c|c|c|}
\hline & $\begin{array}{l}\text { Overall } \\
(n=43)\end{array}$ & $\begin{array}{l}\text { No } \\
\text { Resolution } \\
(n=16)\end{array}$ & $\begin{array}{l}\text { Resolution } \\
(n=27)\end{array}$ & $\begin{array}{l}\text { Log Rank Test P- } \\
\text { value }\end{array}$ \\
\hline MACE, n (\%) & $\begin{array}{l}14 \\
(32.6)\end{array}$ & $9(56.3)$ & 5 (18.5) & 0.005 \\
\hline CV death, $n$ (\%) & $4(9.3)$ & $3(18.8)$ & $1(3.7)$ & 0.062 \\
\hline All-cause death, $n(\%)$ & $7(16.3)$ & $5(31.3)$ & $2(7.4)$ & 0.022 \\
\hline Thrombo-embolic events, n (\%) & $\begin{array}{l}12 \\
(27.9)\end{array}$ & $8(50.0)$ & $4(14.8)$ & 0.008 \\
\hline $\begin{array}{l}\text { Major bleeding (BARC 2/3), n } \\
(\%)\end{array}$ & $4(9.3)$ & $2(12.5)$ & $2(7.4)$ & 0.296 \\
\hline
\end{tabular}


Categorical data are presented as counts and percentages and analyzed using Log rank test. CV death = Cardiovascular death, MACE $=$ Major adverse cardiac events

Table 4: Unadjusted and adjusted effects of LVT resolution on long-term mortality, MACE and thromboembolic events.

\begin{tabular}{|c|c|c|c|c|c|c|}
\hline \multirow[t]{2}{*}{ All-cause death } & \multicolumn{2}{|l|}{ MACE } & \multicolumn{2}{|c|}{ Thrombo- Embolic events } & \multirow[b]{2}{*}{$\mathrm{HR}(95 \% \mathrm{Cl})$} & \multirow[b]{2}{*}{ p-value } \\
\hline & $\mathrm{HR}(95 \% \mathrm{Cl})$ & p-value & $\mathrm{HR}(95 \% \mathrm{Cl})$ & p-value & & \\
\hline $\begin{array}{l}\text { Univariate } \\
0.018\end{array}$ & $0.18(0.03-0.93)$ & 0.041 & $0.26(0.09-0.77)$ & 0.015 & $0.23(0.07-($ & $.78)$ \\
\hline \multicolumn{7}{|l|}{ Multivariate } \\
\hline $\begin{array}{l}\text { Model } 1^{*} \\
0.013\end{array}$ & $0.22(0.04-1.21)$ & 0.081 & $0.24(0.08-0.71)$ & 0.010 & $0.21(0.06$ & $0.72)$ \\
\hline $\begin{array}{l}\text { Model } 2^{\star \star} \\
0.015\end{array}$ & $0.14(0.03-0.75)$ & 0.021 & $0.22(0.07-0.68)$ & 0.008 & $0.22(0.06-($ & $0.75)$ \\
\hline $\begin{array}{l}\text { Model } 3^{\star \star \star} \\
0.029\end{array}$ & $1.10(0.12-9.73)$ & 0.932 & $0.31(0.09-1.03)$ & 0.052 & $0.24(0.07-c$ & $.86)$ \\
\hline
\end{tabular}

Univariate and multivariate Cox proportional hazard models were applied to assess the effect of LVT resolution on all-cause death, MACE and thrombo-embolic events. The $p$ values in bold indicate a value of $<0.05 . \mathrm{Cl}=$ Confidence interval $\mathrm{HR}=$ Hazard ratio .

*Model 1 was adjusted for age and sex.

${ }^{\star *}$ Model 2 was adjusted for STEMI and heart failure.

${ }^{\star \star \star}$ Model 3 was adjusted for Nt-proBNP and CK values.

Table 5: Unadjusted and adjusted effects of TAT with ticagrelor/prasugrel on thrombus resolution.

TAT with ticagrelor/prasugrel 


\begin{tabular}{|c|c|c|}
\hline & $\mathrm{HR}(95 \% \mathrm{Cl})$ & P-value \\
\hline Univariate & $3.67(1.53-8.81)$ & 0.004 \\
\hline \multicolumn{3}{|l|}{ Multivariate } \\
\hline Model $1 *$ & $3.69(1.53-8.91)$ & 0.004 \\
\hline Model 2** & $3.25(1.22-8.68)$ & 0.019 \\
\hline Model $3 * \star \star$ & $2.69(1.10-6.58)$ & 0.030 \\
\hline
\end{tabular}

Univariate and multivariate Cox proportional hazard models were applied to assess the effect of TAT with ticagrelor/prasugrel on LVT resolution. The $p$ values in bold indicate a value of $<0.05$.

$\mathrm{Cl}=$ Confidence interval $\mathrm{HR}=$ Hazard ratio.

*Model 1 was adjusted for age and sex.

${ }^{\star \star}$ Model 2 was adjusted for STEMI and heart failure.

${ }^{* \star *}$ Model 3 was adjusted for Nt-proBNP and CK values.

\section{Figures}
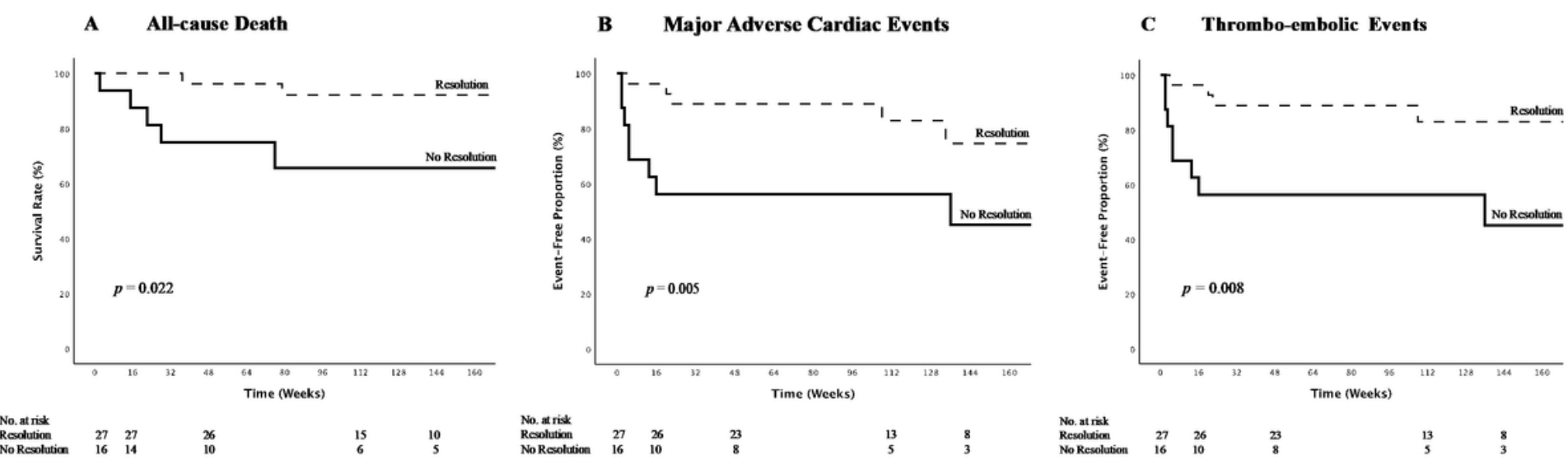

Figure 1

Survival curves of A) All-cause death, B) MACE, and C)Thromboembolic events. 